Materials and Methods We compared the real observed costs incurred by preparing the intravenous mixtures in the pharmacy service and the expected cost if the mixtures were prepared on the wards by using complete vials for each patient and dose, discarding the remainder of the dose.

We have focused the study on the intravenous mixtures area selecting those drugs which need to be prepared individually for the correct dose and those used in the paediatric and neonatology area due to the low dose needed and its variability; however we excluded drugs used in oncology and nutrition from this study.

Results During 2011, 4053 intravenous mixtures were prepared.

The centralised preparation of liposomal amphotericin B (1017 treatments) made an estimated hypothetical saving of $€ 15,226$; infliximab preparation (894) hypothetically saved $€ 122,856$.

Romiplostim (234) generated savings of $€ 59,551$ and tocilizumab (174) €11,280

In the neonatology area the standard preparation of 200 IU epoetin beta from NeoRecormon 500 IU hypothetically saved $€ 603$ with 1623 treatments.

Agalsidase alfa, a high financial impact drug used in Fabry's disease, hypothetically made savings of $€ 62,253$ with 111 preparations.

Total savings generated by centralising the preparation of intravenous mixtures with these 6 drugs amounted to $€ 271,770$.

The median saving exceeded $€ 67 /$ treatment and $€ 744 /$ day. We achieved this situation by sharing vials and using the dose remaining from one treatment to prepare the next one.

Conclusions Centralization of intravenous mixtures allows us to increase efficiency and generate important financial savings, but in addition to increase the quality of healthcare, because it also involves us in pharmacotherapeutic monitoring and avoiding medicines errors. This practise also ensures drugs are handled correctly, which helps maintain their physicochemical and microbiological stability.

No conflict of interest.

\section{OHP-041 FORMULARY DECISION-MAKING FOR BIOSIMILARS: CONSIDERATIONS FOR HOSPITAL PHARMACISTS}

doi:10.1136/ejhpharm-2013-000276.415

'L Green, ${ }^{2}$ Krämer. 'Amgen Inc., Scientific Affairs, Thousand Oaks, USA; 'Johannes Gutenberg-Universität Mainz, Department of Pharmacy, Mainz, Germany

Background It has been 6 years since the first biosimilar was approved for use in the European Union (EU). Given the likelihood that biosimilar monoclonal antibodies will be approved in Europe in the near future, it is timely to review the formulary selection criteria for biologicals and biosimilars.

The European Medicines Agency (EMA) has issued guidelines that define the regulation of biosimilars in Europe and recommend approaches to establish biosimilarity. However, several questions regarding the assessment of biosimilars for formulary inclusion remain unanswered, including those related to manufacturing and drug supply.

Purpose To aid hospital pharmacists in developing evaluation criteria for biosimilars under consideration for formulary inclusion.

Materials and Methods EU and United States biosimilar legislation, peer-reviewed literature, public data, EMA guidelines and formulary decision-making practises were reviewed to identify key considerations and evaluation criteria for including biosimilars in a formulary.

Results Biosimilars may differ in certain characteristics from their reference product and, therefore, require more extensive evaluation during formulary consideration than small-molecule generics. Recent drug shortages and stockouts throughout Europe underscore the need to evaluate manufacturer reliability and supply chain considerations in formulary reviews of biosimilars. Indications approved for the reference product may not be approved for the biosimilar and should be considered during formulary review. Therefore, we propose a checklist that includes criteria for product evaluation and manufacturer-related parameters, such as differences in administration devices, drug availability, inventory turns, history of shortages, recalls, inventory levels, manufacturing redundancy and supply chain security.

Conclusions Ensuring a stable, reliable supply of quality products is a critical component of healthcare. Product, manufacturer, and pharmacoeconomic information should be considered in formulary decision-making for biosimilars. A checklist of key product- and manufacturer-related information will be promoted thorough evaluation of biosimilars, permitting educated decisions regarding formulary inclusion.

No conflict of interest.

\section{OHP-042 HEPARIN-INDUCED THROMBOCYTOPENIA (HIT): PRE-TEST CLINICAL SCORE (4TS) TO JUSTIFY DANAPAROID PRESCRIPTIONS? WHAT ELSE?}

doi:10.1136/ejhpharm-2013-000276.416

G Baussant, F Loeuillet-Moreau, A Le Joubioux, B Frimat. Lens Hospital Center, Pharmacy, Lens, France

Background HIT is a prothrombotic adverse drug reaction caused by heparin and requires an alternative anticoagulant: danaparoid. Because of its cost and the specific indication, the physicians must request two laboratory tests with prescriptions (LT: Platelet Aggregation Test, Anti PF4H) and a 4Ts assessment, in order to have danaparoid dispensed.

Purpose To find out whether prescriptions are justified and if we can use the 4Ts score as a basis for HIT detection.

Materials and Methods We analysed 5 years of prescriptions: 4Ts score results (the 4Ts assessment is used to arrive at a high (score 6 or more), intermediate (score 4-5) or low (score 3 or less) probability of HIT.

Of 72 hospitalised patients followed (LT and/or prescription), 34 had a LT score without danaparoid prescription (32 negative and 2 positive results). 38 had a prescription that had been dispensed. 32 patients of these 38 had a 4Ts score. Looking at the 4 Ts' results:

- $3.12 \%(1 / 32)$ patients had low score (LT not requested).

- $62.5 \%(20 / 32)$ came into the intermediate category (LT: 8/20 negative $-4 / 20$ positive - uncertain $3 / 20$ - not requested $5 / 20)$.

- $34.4 \%(11 / 32)$ came into the high-score group (LT: $4 / 11$ negative $-4 / 11$ positive $-1 / 11$ uncertain - not requested $2 / 11$ ).

In $60.5 \%$ of the cases $(23 / 38)$, the prescription was justified by a high score or a positive LT test or HIT diagnosed before. In $39.5 \%$ of the cases (15/38), a danaparoid prescription wasn't justified: 7 patients still received danaparoid after negative LT results and 8 without a 4Ts score.

Conclusions In our hospital, positive predictive value doesn't match like it's written in the literature. The 4Ts score doesn't seem to favourably correspond with HIT laboratory testing results. A new scoring HIT Expert Probability Score is right now in validation. Will it be more suitable for our practise?

No conflict of interest.

\section{OHP-043 HIP AND KNEE PROSTHESES IN REPLACEMENT SURGERY: REVIEW OF USE}

doi:10.1136/ejhpharm-2013-000276.417

'A Luna Higuera, 'M Toca Muñoz, ${ }^{21}$ Martín Rodríguez, 'S García Agudo, 'IM Muñoz Castillo. 'HRU Carlos Haya, Pharmacy, Malaga, Spain; ${ }^{2}$ HRU Carlos Haya, Traumatology and Orthopaedic Surgery, Malaga, Spain 